\title{
An Holistic State Equation for Timed Petri Nets
}

\author{
Matthias Werner ${ }^{1}$, Louchka Popova-Zeugmann ${ }^{2}$, Mario Haustein ${ }^{1}$, and E. Pelz ${ }^{3}$ \\ 1 Professur Betriebssysteme, Technische Universität Chemnitz \\ 2 Institut für Informatik, Humboldt-Universität zu Berlin \\ 3 LACL, UPEC, France
}

\section{Introduction}

Timed Petri nets (TPN) or Duration Petri nets (DPN) is a well-know approach to extend "classic" Petri nets in order to allow the modeling of time [1].

In [2], a state equation for TPN was provided that describes the net's marking in an algebraic manner, but not its transitions clocks. Hence, proofing the nonreachability of a marking is mainly done by symbolic manipulation of the state equation, which is impractical for the automated generation of such proofs. Here, we introduce a holistic state equation that allows for modeling the clocks algebraically in addition to markings and thus provides a more automatical way to show the nonreachablity of specific markings.

\section{Timed Petri Nets}

\subsection{Notation}

This section introduces the basic notations we use in our paper. $\mathbb{N}^{+}=\mathbb{N} \backslash\{0\}$ denotes the set of natural numbers without 0 , and $\mathbb{Q}_{0}^{+}$denotes the set of nonnegative rational numbers. Let $S$ be a finite set. $|S|$ is the number of elements of $S$. Multisets can contain an element multiple times and are designated by Fraktur letters. The $\uplus$-operator denotes the union of multisets. The number of occurrences of each element in the result of the $\uplus$-operation is given by the sum of the occurrences of this element in both operands. $|\mathfrak{S}|_{e}$ denotes the multiplicity of $e$ in the multiset $\mathfrak{S} . \mathbb{1}_{c}$ is the indicator function which yields 1 iff the condition $c$ holds or 0 otherwise.

A matrix $\mathbf{A} \in \mathcal{M}(m, n)$ is a matrix with $m$ rows and $n$ columns. A superindex in parentheses distinguishes different matrices or vectors, and their elements, respectively. $\mathbf{Z}_{m \times n}=\left(z_{i, j}\right) \in \mathcal{M}(m, n)$ is the zero matrix with $z_{i, j}=0$ and $\mathbf{E}_{n}=\left(e_{i, j}\right) \in \mathcal{M}(n, n)$ denotes the identity matrix with:

$$
e_{i, j}= \begin{cases}1 & i=j \\ 0 & \text { else }\end{cases}
$$


The relation $\boldsymbol{r}^{(1)} \leq \boldsymbol{r}^{(2)}$ of the two vectors $\boldsymbol{r}^{(1)}, \boldsymbol{r}^{(2)} \in \mathcal{M}(m, 1)$ means, that all elements of $\boldsymbol{r}_{i}^{(1)}$ are less or equal than the corresponding elements of $\boldsymbol{r}_{i}^{(2)}$. The relation $\boldsymbol{r}^{(1)} \mathbb{\leq} \boldsymbol{r}^{(2)}$ means, that the above relation does not hold, i.e. there exists at least one $i \in\{1, \ldots, m\}$ with $\boldsymbol{r}_{i}^{(1)}>\boldsymbol{r}_{i}^{(2)}$. The relations $<$ and $\nless$ are defined analogously.

\subsection{Timed Petri Nets}

Definition 1 (Petri net). The structure $\mathcal{N}=\left(P, T, F, V, m^{(0)}\right)$ is called a Petri net (PN), iff

1. $P, T$ are finite sets with $P \cap T=\emptyset$ and $P \cup T \neq \emptyset$,

2. $F \subseteq(P \times T) \cup(T \times P)$ (relation between places and transitions),

3. $V: F \rightarrow \mathbb{N}^{+}$(weight of the arcs),

4. $m^{(0)}: P \rightarrow \mathbb{N}$ (initial marking)

A marking of a Petri net is a function $m: P \rightarrow \mathbb{N}$, such that $m(p)$ denotes the number of tokens at the place $p$. The pre- and post-sets of a transition $t$ are given by $\bullet t=\{p:(p, t) \in F\}$ and $t \bullet=\{p:(t, p) \in F\}$, respectively. Each transition $t \in T$ induces the marking change $t^{-}$and $t^{+}$, defined as follows:

$$
t^{-}(p)=\left\{\begin{array}{ll}
V(p, t) & (p, t) \in F \\
0 & \text { else }
\end{array} \quad t^{+}(p)= \begin{cases}V(t, p) & (t, p) \in F \\
0 & \text { else }\end{cases}\right.
$$

A transition $t \in T$ is enabled (may fire) at a marking $m$, iff $t^{-}(p) \leq m(p)$ for every place $p \in P$. When an enabled transition $t$ at a marking $m$ fires, this yields a new marking $m^{\prime}$ given by $m^{\prime}(p):=m(p)-t^{-}(p)+t^{+}(p)$. The firing is denoted by $m \stackrel{t}{\rightarrow} m^{\prime}$.

\section{Definition 2 (Timed Petri net).}

The structure $\mathcal{Z}=(\mathcal{N}, D)$ is called a Timed Petri net (TPN) iff:

1. $\mathcal{N}$ (called Skeleton of $\mathcal{Z}$ ) is a Petri net,

2. $D: T \rightarrow \mathbb{Q}_{0}^{+}$.

$D(t)$ is the duration of the firing transition $t$ and denotes the delay of $t$. It is easy to see, that considering TPNs with $D: T \rightarrow \mathbb{N}$ will not result in a loss of generality. Therefore, only such time functions $D$ will be considered subsequently.

An active transition $t$ passes through three phases. First it consumes tokens from $\bullet t$ which leads to a new marking $m^{\prime}(p):=m(p)-t^{-}(p)$. This change takes no time. Then time $D(t)$ passes. During this time, the marking $m^{\prime}$ may change to $m^{\prime \prime}$ by the firing of other transitions. Finally $t$ delivers tokens to t• which leads to the marking $m^{\prime \prime \prime}(p):=m^{\prime \prime}(p)+t^{+}(p)$.

Definition 3 (Maximal step).

Let $z=(m, u)$ be a state in the Timed Petri net $\mathcal{Z}=\left(P, T, F, V, m_{0}, D\right)$. Then $M \subseteq T$ is a maximal step in $z$, if 
1. $\forall t \in M: u(t)=0$,

2. $\sum_{t \in M} t^{-} \leq m$,

3. $\forall \hat{t} \in T \backslash M: \hat{t}^{-} \leq m \wedge u(\hat{t})=0 \Longrightarrow \hat{t}^{-} \not \leq m-\sum_{t \in M} t^{-}$.

In a Timed Petri net, an enabled transition must fire immediately. In case of nonself-concurrent transitions, a transition can only be enabled, if it is not active at the moment. Please note, immediate transitions are implicitly self-concurrent. In the following we will consider all delayed transitions as not self-concurrent.

\section{Definition 4 (Firing).}

Let $z_{1}=\left(m_{1}, u_{1}\right)$ be a state in the Timed Petri net $\mathcal{Z}$ and $M \subseteq T$. Then $M$ can fire in $z_{1}$ (notation: $z_{1} \stackrel{M}{\longrightarrow}$ ), if $M$ is a maximal step in $z_{1}$. After the firing of $M$ the net $\mathcal{Z}$ changes into the state $z_{2}=\left(m_{2}, u_{2}\right)$ (notation: $z_{1} \stackrel{M}{\longrightarrow} z_{2}$ ) with:

$$
m_{2}:=m_{1}-\sum_{t \in M} t^{-}+\sum_{\substack{t \in M \\ D(t)=0}} t^{+} \quad \text { and } \quad u_{2}(t):= \begin{cases}D(t) & t \in M \\ u_{1}(t) & \text { else }\end{cases}
$$

In a Timed Petri net it is possible that after firing of a maximal step containing transitions with zero delay some transitions are still enabled. For that purpose, we define a global step:

\section{Definition 5 (Global step).}

Let $z$ be a state in the Timed Petri net $\mathcal{Z}$. The multiset $\mathfrak{G}$ over $T$ is called a global step in $z$, that is computed by the following procedure:

1. $\mathfrak{G}:=\emptyset$;

2. Let $M$ be a maximal step in $z$;

3. if $M \neq \emptyset$ then $\mathfrak{G}:=\mathfrak{G} \uplus M$ else stop;

4. Let $z \stackrel{M}{\longrightarrow} z^{\prime}$; Set $z:=z^{\prime}$; goto 2 ;

To ensure finite global steps, we do not allow Timed Petri nets with time deadlocks, i.e., with a closed directed path that contains immediate transitions only.

\section{Definition 6 (Elapsing of time).}

Let $z_{1}=\left(m_{1}, u_{1}\right)$ be a state in the Timed Petri net $\mathcal{Z}$. Then, the elapsing of one time unit is possible in $\mathcal{Z}$ (notation: $z_{1} \rightarrow \underset{1}{\rightarrow}$ ), if

$$
\forall t \in T: u_{1}(t)=0 \Longrightarrow t^{-} \not \leq m_{1}
$$

After the elapsing of one time unit the Timed Petri net $\mathcal{Z}$ is in the state $z_{2}=$ $\left(m_{2}, u_{2}\right)$ (notation: $z_{1} \underset{1}{\rightarrow} z_{2}$ ) with:

$$
m_{2}:=m_{1}+\sum_{\substack{t \in T \\ u_{1}(t)=1}} t^{+} \quad \text { and } \quad u_{2}(t):= \begin{cases}u_{1}(t)-1 & u_{1}(t) \geq 1 \\ 0 & \text { else }\end{cases}
$$




\section{State Equation}

\subsection{Structure}

Like in classical Petri nets, we describe the structure of a Timed Petri net by two matrices $\mathbf{C}^{+}, \mathbf{C}^{-} \in \mathcal{M}(|P|,|T|)$ over the base set $\mathbb{N}$, with $c_{i, j}^{+}=t_{j}^{+}\left(p_{i}\right)$ and $c_{i, j}^{-}=t_{j}^{-}\left(p_{i}\right)$. Furthermore the delay of each transition is encoded in a delay matrix $\boldsymbol{\Gamma} \in \mathcal{M}(|T|, d)$, where $d=\max \{D(t): t \in T\}+1$ specifies the maximum delay. The matrix $\boldsymbol{\Gamma}$ is given by the following definition:

$$
\gamma_{i, j}= \begin{cases}1 & j=D\left(t_{i}\right)+1 \\ 0 & \text { else }\end{cases}
$$

\subsection{Dynamics}

The dynamic of a Petri net at each point of time, is unambiguously decribed by its state. In our model, the state consists of two parts, the place marking $\boldsymbol{m}$ and the clock matrix $\mathbf{U}$. The place marking is given by a vector $\boldsymbol{m} \in \mathcal{M}(|P|, 1)$ over $\mathbb{N}$, which specifies how many tokens are on each place. In contrast to classical Petri nets not only the marking is part of the state. The clock matrix $\mathbf{U} \in \mathcal{M}(|T|, d)$ accounts for all active transitions. The element $u_{i, j}$ specifies how often a transition $t_{i}$ has consumed $D\left(t_{i}\right)-j+1$ time steps ago, and therefore how often it will deliver in $j-1$ time steps from now. Thus, the first row of $\mathbf{U}$ states how many times each transition will finish right now. Because a zero delay transition $t_{k}$ is intrinsically self-concurrent, the value of $u_{k, 1}$ may have any value from $\mathbb{N}$. But the row sum of delayed transitions is at most 1 , due to the lack of self-concurrency. It is easy to see, that all values $u_{i, j}$ for $j>D\left(t_{i}\right)+1$ are zero.

To calculate the state reached by a given firing sequence, we have to represent the sequence inside our equation. In classical Petri nets this is done by the Parikh vector. We extend the Parikh vector to the Parikh matrix. The Parikh matrix $\Psi \in \mathcal{M}(|T|,|T|)$ of a global step $\mathfrak{G}$ is defined by

$$
\boldsymbol{\Psi}=\operatorname{diag}\left(\psi_{1}, \ldots, \psi_{|T|}\right) \quad \text { with } \quad \psi_{i}=|\mathfrak{G}|_{t_{i}}
$$

To use $\boldsymbol{m}, \mathbf{U}$ and $\boldsymbol{\Psi}$ together, three operators $\mathbf{A} \in \mathcal{M}(d, 1)$ (adoption operator), $\mathbf{R} \in \mathcal{M}(d, d)$ (progress operator) and $\boldsymbol{\Lambda} \in \mathcal{M}(|T|, d)$ (selection operator) are necessary, specified by the corresponding matrices:

$$
\mathbf{A}=\left(\begin{array}{llll}
1 & 0 & \ldots & 0
\end{array}\right)^{\mathrm{T}} \quad r_{i, j}=\left\{\begin{array}{ll}
1 & i-j=1 \\
0 & \text { else }
\end{array} \quad \lambda_{i, j}= \begin{cases}1 & j=1 \\
0 & \text { else }\end{cases}\right.
$$

$\dot{\boldsymbol{\Psi}}=\boldsymbol{\Psi} \cdot \boldsymbol{\Lambda} \cdot \mathbf{A}$ gains the "classical" Parikh vector. The term $\overline{\boldsymbol{\Psi}}^{(i)}$ later used is an abbreviation of $\sum_{j=1}^{i} \dot{\boldsymbol{\Psi}}^{(j)}$ and specifies how often each transition has fired until time step $i$. 


\subsection{Algebraical Representation of State Changes}

Using the definitions of the section above, we can derive an algebraic description for a single state change:

$$
\begin{aligned}
& \mathbf{U}^{\prime}=\mathbf{U}+\boldsymbol{\Psi} \boldsymbol{\Gamma} \quad \boldsymbol{m}^{\prime}=\boldsymbol{m}-\mathbf{C}^{-} \boldsymbol{\Psi} \boldsymbol{\Lambda} \mathbf{A}+\mathbf{C}^{+} \boldsymbol{\Psi} \boldsymbol{\Gamma} \mathbf{A} \quad \text { (Firing) } \\
& \mathbf{U}^{\prime \prime}=\mathbf{U}^{\prime} \mathbf{R} \quad \boldsymbol{m}^{\prime \prime}=\boldsymbol{m}^{\prime}+\mathbf{C}^{+} \mathbf{U}^{\prime} \mathbf{R} \mathbf{A} \quad \text { (Time elapsing) }
\end{aligned}
$$

To apply Equations (1) and (2) to arbitrary sequences, we introduce time indices to $\boldsymbol{m}$ and $\mathbf{U}$ and mark the results of Equation (1) by a hat and the results of Equation (2) by a tilde. Firing steps and timing steps are dennoted by

$$
\left(\tilde{\boldsymbol{m}}^{(i)}, \tilde{\mathbf{U}}^{(i)}\right) \stackrel{\mathfrak{G}^{(i)}}{\longrightarrow}\left(\hat{\boldsymbol{m}}^{(i)}, \hat{\mathbf{U}}^{(i)}\right) \quad \text { and } \quad\left(\hat{\boldsymbol{m}}^{(i)}, \hat{\mathbf{U}}^{(i)}\right) \underset{1}{\longrightarrow}\left(\tilde{\boldsymbol{m}}^{(i+1)}, \tilde{\mathbf{U}}^{(i+1)}\right)
$$

respectively. Every firing sequence $\sigma$ can then be represented by alternating firing and time elapsing steps, where some of the $\mathfrak{G}$ can be empty sets of course:

$$
\sigma:\left(\tilde{\boldsymbol{m}}^{(1)}, \tilde{\mathbf{U}}^{(1)}\right) \stackrel{\mathfrak{G}^{(1)}}{\longrightarrow}\left(\hat{\boldsymbol{m}}^{(1)}, \hat{\mathbf{U}}^{(1)}\right) \underset{1}{\longrightarrow} \cdot \stackrel{\mathfrak{G}^{(2)}}{\longrightarrow} \cdots \underset{1}{\longrightarrow} \cdot \stackrel{\mathfrak{G}^{(i)}}{\longrightarrow}\left(\hat{\boldsymbol{m}}^{(i)}, \hat{\mathbf{U}}^{(i)}\right)
$$

The term $\tilde{\boldsymbol{m}}^{(1)}$ is equal to the initial marking $\boldsymbol{m}^{(0)}$ and $\tilde{\mathbf{U}}^{(1)}$ is the zero matrix $\mathbf{Z}_{|T| \times d}$, because no transition is active in the initial state.

\subsection{State equation}

We now consider the actual state equation. From Equations (1) and (2) we can derrive the following equations on $\hat{\boldsymbol{m}}^{(i)}$ and $\hat{\mathbf{U}}^{(i)}$ for the sequence $\sigma$.

$$
\begin{aligned}
\hat{\mathbf{U}}^{(i)} & =\sum_{j=1}^{i} \boldsymbol{\Psi}^{(j)} \boldsymbol{\Gamma} \mathbf{R}^{i-j} \\
\hat{\boldsymbol{m}}^{(i)} & =\boldsymbol{m}^{(0)}+\mathbf{C}^{+}\left(\sum_{j=1}^{i} \boldsymbol{\Psi}^{(j)} \boldsymbol{\Gamma} \sum_{k=0}^{i-j} \mathbf{R}^{k}\right) \mathbf{A}-\mathbf{C}^{-} \overline{\boldsymbol{\Psi}}^{(i)} \\
& =\boldsymbol{m}^{(0)}+\mathbf{C}^{+}\left(\psi_{k}^{\left(i-D\left(t_{k}\right)\right)}+\cdots+\psi_{k}^{(1)}\right)_{k}-\mathbf{C}^{-} \overline{\boldsymbol{\Psi}}^{(i)}
\end{aligned}
$$

With defining $\underline{\boldsymbol{\Psi}}^{(i)}:=\left(\psi_{k}^{\left(i-D\left(t_{k}\right)\right)}+\cdots+\psi_{k}^{(1)}\right)_{k}$, Equation (4) can be expressed in a more compact way:

$$
\hat{\boldsymbol{m}}^{(i)}=\boldsymbol{m}^{(0)}+\mathbf{C}^{+} \underline{\boldsymbol{\Psi}}^{(i)}-\mathbf{C}^{-} \overline{\boldsymbol{\Psi}}^{(i)}
$$

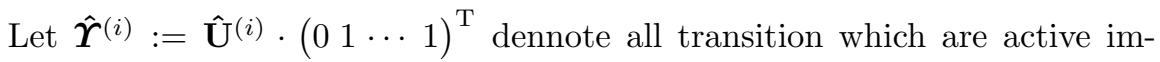
mediately after the zero delay transitions in firing step $i$ have delivered. Since each delayed transition can only be active once at each point of time, only clock matrices $\hat{\mathbf{U}}^{(i)}$ are valid, which fulfill the following constraint:

$$
(1 \cdots 1)^{\mathrm{T}} \stackrel{!}{\geq} \hat{\mathbf{U}}^{(i)} \cdot\left(\begin{array}{llll}
0 & 1 & \cdots & 1
\end{array}\right)^{\mathrm{T}}=\hat{\boldsymbol{\Upsilon}}^{(i)}
$$


By substituting $\hat{\mathbf{U}}^{(i)}$ in the former equation with the right side of Equation (3), we get:

$$
(1 \cdots 1)^{\mathrm{T}} \stackrel{!}{\geq}\left(\psi_{k}^{\left(i-D\left(t_{k}\right)+1\right)}+\cdots+\psi_{k}^{(i)}\right)_{k}=\hat{\boldsymbol{\Upsilon}}^{(i)}
$$

Because the inner term of the former equation is zero for immediate transitions, it follows that $\hat{\boldsymbol{\Upsilon}}^{(i)}$ must be an element of $B_{1} \times \cdots \times B_{|T|}$, with

$$
B_{k}= \begin{cases}\{0,1\} & D\left(t_{k}\right)>0 \\ \{0\} & \text { else }\end{cases}
$$

The right part of Equation (6) helps reformulating Equation (4) into

$$
\hat{\boldsymbol{m}}^{(i)}=\boldsymbol{m}^{(0)}+\left(\mathbf{C}^{+}-\mathbf{C}^{-}\right) \cdot \underline{\boldsymbol{\Psi}}^{(i)}-\mathbf{C}^{-} \hat{\boldsymbol{\Upsilon}}^{(i)}
$$

The vector $\underline{\Psi}^{(i)}$ sums up all transition which have completed until time step $i$. It is easy to see that $\underline{\boldsymbol{\Psi}}^{(i)}+\hat{\boldsymbol{\Upsilon}}^{(i)}$ yields the Parikh vector $\overline{\boldsymbol{\Psi}}^{(i)}$. An equation for $\tilde{\boldsymbol{m}}^{(i)}$ can be obtained by applying Equations (2) to Equation (7):

$$
\begin{aligned}
& \tilde{\boldsymbol{m}}^{(i)}=\hat{\boldsymbol{m}}^{(i-1)}+\mathbf{C}^{+} \hat{\mathbf{U}}^{(i-1)} \mathbf{R} \mathbf{A} \\
& =\hat{\boldsymbol{m}}^{(i-1)}+\mathbf{C}^{+} \hat{\mathbf{U}}^{(i-1)} \cdot\left(\begin{array}{lllll}
0 & 1 & 0 & \cdots & 0
\end{array}\right)^{\mathrm{T}} \\
& =\hat{\boldsymbol{m}}^{(i-1)}+\mathbf{C}^{+} \hat{\mathbf{U}}^{(i-1)} \cdot\left(\begin{array}{lllll}
0 & 1 & 1 & \cdots & 1
\end{array}\right)^{\mathrm{T}}-\mathbf{C}^{+} \hat{\mathbf{U}}^{(i-1)} \cdot\left(\begin{array}{lllll}
0 & 0 & 1 & \cdots & 1
\end{array}\right)^{\mathrm{T}} \\
& =\hat{\boldsymbol{m}}^{(i-1)}+\mathbf{C}^{+} \hat{\mathbf{U}}^{(i-1)} \cdot\left(\begin{array}{llll}
0 & 1 & \cdots & 1
\end{array}\right)^{\mathrm{T}}-\mathbf{C}^{+} \tilde{\mathbf{U}}^{(i)} \cdot\left(\begin{array}{llll}
0 & 1 & \cdots & 1
\end{array}\right)^{\mathrm{T}} \\
& =\hat{\boldsymbol{m}}^{(i-1)}+\mathbf{C}^{+} \hat{\boldsymbol{\Upsilon}}^{(i-1)}-\mathbf{C}^{+} \tilde{\boldsymbol{\Upsilon}}^{(i)} \\
& =\boldsymbol{m}^{(0)}+\left(\mathbf{C}^{+}-\mathbf{C}^{-}\right) \cdot\left(\underline{\Psi}^{(i-1)}+\hat{\boldsymbol{\Upsilon}}^{(i-1)}\right)-\mathbf{C}^{+} \tilde{\boldsymbol{\Upsilon}}^{(i)}
\end{aligned}
$$

The vector $\tilde{\boldsymbol{\Upsilon}}^{(i)}:=\tilde{\mathbf{U}}^{(i)} \cdot\left(\begin{array}{llll}0 & 1 & \cdots & 1\end{array}\right)^{\mathrm{T}}$ specifies all transition which are in progress after the $i$-th time step has finished. This vector is elementwise less or equal than $\hat{\boldsymbol{\Upsilon}}^{(i-1)}$, because we have to subtract the elements of the second column of $\hat{\mathbf{U}}^{(i-1)}$ to yield $\tilde{\boldsymbol{\Upsilon}}^{(i)}$. Furthermore $\tilde{\boldsymbol{\Upsilon}}^{(i)}$ is elementwise less or equal than $\hat{\boldsymbol{\Upsilon}}^{(i)}$, which follows directly from Equation (1). Obviously no element of the $\boldsymbol{\Upsilon}$-vectors can be negative. In case of a transition $t_{k}$ with delay less than 2 , the $k$-th element of $\tilde{\boldsymbol{\Gamma}}^{(i)}$ is zero in any case, because due to the construction of $\boldsymbol{\Gamma}$ only the first or the second element of the $k$-th row of $\hat{\boldsymbol{U}}^{(i-1)}$ can be non-zero. Consequently it holds:

$$
(0)_{k} \stackrel{!}{\leq} \tilde{\boldsymbol{\Upsilon}}^{(i)} \stackrel{!}{\leq}\left\{\begin{array}{c}
\hat{\boldsymbol{\Upsilon}}^{(i-1)} \\
\hat{\boldsymbol{\Upsilon}}^{(i)}
\end{array}\right\} \stackrel{!}{\leq}\left(\mathbb{1}_{D\left(t_{k}\right)>0}\right)_{k} \quad \text { and } \quad \tilde{\boldsymbol{\Upsilon}}^{(i)} \stackrel{!}{\leq}\left(\mathbb{1}_{D\left(t_{k}\right)>1}\right)_{k}
$$

\section{Non-Reachability and Application Example}

State equations provide a criterion to decide whether a given marking is not reachable in a specified Petri net. When the state equation does not have a solution, the marking is not reachable. In case of Timed Petri nets, Equations (7) 
and (9) as well es the constraint Equations (10) form a system of diophantene (in-)equalities. If this system does not have a valid solution, a given $\hat{\boldsymbol{m}}$-marking and $\tilde{\boldsymbol{m}}$-marking, resp., is not reachable. A solution is valid if and only if the Parikh vector candidate does not contain a negative element. Furthermore we can draft on predecessor markings and maximal step conditions to further discard some valid solutions of the state equation.

In this section we show how the state equation in the recent section can be used for a more systematical nonreachability proof of the example given in [2]. Consider the following Timed Petri net $\mathcal{Z}$ :

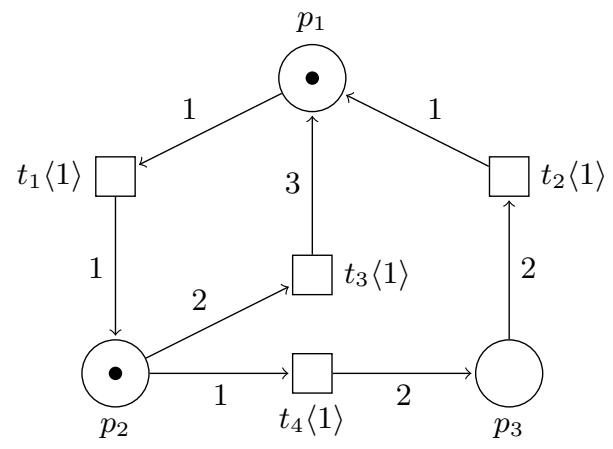

Fig. 1. Petri net from [2]; transition times are given in angle bracket

We want to show that the marking $\boldsymbol{m}^{*}=\left(\begin{array}{lll}0 & 2 & 0\end{array}\right)^{\mathrm{T}}$ is not reachable. To do so, one has to show that both $\tilde{\boldsymbol{m}}^{(i)}=\boldsymbol{m}^{*}$ and $\hat{\boldsymbol{m}}^{(i)}=\boldsymbol{m}^{*}$ are not reachable in $\mathcal{Z}$. Lets consider the $\tilde{\boldsymbol{m}}^{(i)}$-case first. First we have to determine all $\boldsymbol{\Psi}^{(i-1)}+\hat{\boldsymbol{\Upsilon}}^{(i-1)}$ which solve Equation $(9)$. The solution space $\mathcal{S}_{t}$ depends on $\tilde{\boldsymbol{\Upsilon}}^{(i)}$ and can be calculated by the GAUSS-Algorithm:

$$
\mathcal{S}_{t}\left(\tilde{\boldsymbol{\Upsilon}}^{(i)}\right)=\left\{\left(\begin{array}{r}
-1 \\
0 \\
0 \\
0
\end{array}\right)+\left(\begin{array}{rrrr}
3 & 2 & 6 & 2 \\
0 & 0 & 0 & -1 \\
1 & 1 & 3 & 1 \\
0 & 0 & 0 & 0
\end{array}\right) \cdot \tilde{\boldsymbol{\Upsilon}}^{(i)}+k \cdot\left(\begin{array}{l}
1 \\
1 \\
0 \\
1
\end{array}\right): k \in \mathbb{Z}\right\}
$$

In this special case it follows from Equation (10) that $\tilde{\boldsymbol{\Upsilon}}^{(i)}$ must be the zero vector, so only one solution space has to be considered during the further calculation. As stated, a valid solution cannot contain a negative component. Thus, we can rule out all $\hat{\boldsymbol{\Upsilon}}^{(i-1)}$ for which the set $\mathbb{N}^{|T|} \cap\left\{\boldsymbol{S}-\hat{\boldsymbol{\Upsilon}}^{(i-1)}: \boldsymbol{S} \in \mathcal{S}_{t}\right\}$ is empty. Algorithmically this problem can be decided by integer linear programming techniques. In our example at least $\tilde{v}_{3}^{(i-1)}$ must be zero, which narrows the set of possible $\hat{\Upsilon}^{(i-1)}$ down to eight distinct cases, shown in the following table. With the aid of Equation (8), we can calculate the corresponding $\hat{\boldsymbol{m}}^{(i-1)}$ : 


\begin{tabular}{l|l}
$\begin{array}{l}\text { candidates for } \\
\hat{\boldsymbol{\Upsilon}}^{(i-1)}\end{array}$ & $\left(\begin{array}{l}0 \\
0 \\
0 \\
0\end{array}\right)\left(\begin{array}{l}1 \\
0 \\
0 \\
0\end{array}\right)\left(\begin{array}{l}0 \\
1 \\
0 \\
0\end{array}\right)\left(\begin{array}{l}1 \\
1 \\
0 \\
0\end{array}\right)\left(\begin{array}{l}0 \\
0 \\
0 \\
1\end{array}\right)\left(\begin{array}{l}1 \\
0 \\
0 \\
1\end{array}\right)\left(\begin{array}{l}0 \\
1 \\
0 \\
1\end{array}\right)\left(\begin{array}{l}1 \\
1 \\
0 \\
1\end{array}\right)$ \\
\hline $\begin{array}{l}\text { corresp. } \\
\hat{\boldsymbol{m}}^{(i-1)}\end{array}$ & $\left(\begin{array}{l}0 \\
2 \\
0\end{array}\right)\left(\begin{array}{l}0 \\
1 \\
0\end{array}\right)\left(\begin{array}{r}-1 \\
2 \\
0\end{array}\right)\left(\begin{array}{r}-1 \\
1 \\
0\end{array}\right)\left(\begin{array}{r}0 \\
2 \\
-2\end{array}\right)\left(\begin{array}{r}0 \\
1 \\
-2\end{array}\right)\left(\begin{array}{r}-1 \\
2 \\
-2\end{array}\right)\left(\begin{array}{r}-1 \\
1 \\
-2\end{array}\right)$
\end{tabular}

Because places cannot contain a negative amount of tokens, we can rule out the last six cases. The remaining two cases can be discarded by aid of Definition 3. According to the definition of a maximal step for all active transitions $t_{k}$, i.e., all transitions $t_{k}$ for which $\hat{v}_{k}=0$, it must hold $\hat{\boldsymbol{m}} \geq t_{k}^{-}$. Now we consider $t_{4}$ which is active in both cases. The maximal step condition $\hat{\boldsymbol{m}}^{(i-1)} \nsucceq\left(\begin{array}{lll}0 & 1 & 0\end{array}\right)^{\mathrm{T}}$ resulting from $t_{4}$ is not fulfilled. Thus, the two remaining solutions are not valid in $\mathcal{Z}$. Consequently $\boldsymbol{m}^{*}$ cannot be reached as a $\tilde{\boldsymbol{m}}$-marking.

Now, we show $\boldsymbol{m}^{*}$ is not reachable as a $\hat{\boldsymbol{m}}$-marking. First, we calculate the set $\mathcal{Y}$ of possible $\hat{\boldsymbol{\Upsilon}}^{(i)}$ by using the definition of the maximal step:

$$
\mathcal{Y}\left(\hat{\boldsymbol{m}}^{(i)}\right)=\left\{\hat{\boldsymbol{\Upsilon}}:\left(\forall k: \hat{v}_{k}=0 \Longrightarrow \hat{\boldsymbol{m}}^{(i)} \nsupseteq t_{k}^{-}\right)\right\}
$$

In our example, at least $\hat{v}_{3}^{(i)}=1$ and $\hat{v}_{4}^{(i)}=1$, otherwise the maximal step condition for $t_{3}$ and $t_{4}$, resp., would not be fulfilled. Then we calculate the solution set $\mathcal{S}_{f}$ of Equation (7).

$$
\mathcal{S}_{f}\left(\hat{\boldsymbol{\Upsilon}}^{(i)}\right)=\left\{\left(\begin{array}{r}
-1 \\
0 \\
0 \\
0
\end{array}\right)+\left(\begin{array}{rrrr}
2 & 2 & 6 & 3 \\
0 & -1 & 0 & 0 \\
1 & 1 & 2 & 1 \\
0 & 0 & 0 & 0
\end{array}\right) \cdot \tilde{\boldsymbol{\Upsilon}}^{(i)}+k \cdot\left(\begin{array}{l}
1 \\
1 \\
0 \\
1
\end{array}\right): k \in \mathbb{Z}\right\}
$$

In the example every possible $\hat{\boldsymbol{\Upsilon}}^{(i)}$ yields at least one Parikh vector $\underline{\boldsymbol{\Psi}}^{(i)}$. From $\hat{\boldsymbol{m}}^{(i)}$ we can calculate $\tilde{\boldsymbol{m}}^{(i)}$ by:

$$
\tilde{\boldsymbol{m}}^{(i)}=\hat{\boldsymbol{m}}^{(i)}+\mathbf{C}^{+} \tilde{\boldsymbol{\Upsilon}}^{(i)}-\mathbf{C}^{+} \hat{\boldsymbol{\Upsilon}}^{(i)}
$$

Due to Equation (10) the vector $\tilde{\boldsymbol{\Upsilon}}^{(i)}$ must be the zero vector in this example. Consequently we can calculate $\tilde{\boldsymbol{m}}^{(i)}$ without further case discriminations on $\tilde{\boldsymbol{\Upsilon}}^{(i)}$ :

$$
\begin{array}{l|l}
\hat{\boldsymbol{\Upsilon}}^{(i)} & \left(\begin{array}{l}
0 \\
0 \\
1 \\
1
\end{array}\right)\left(\begin{array}{l}
1 \\
0 \\
1 \\
1
\end{array}\right)\left(\begin{array}{l}
0 \\
1 \\
1 \\
1
\end{array}\right)\left(\begin{array}{l}
1 \\
1 \\
1 \\
1
\end{array}\right) \\
\hline \text { corresp. } \tilde{\boldsymbol{m}}^{(i)} & \left(\begin{array}{r}
-3 \\
2 \\
-2
\end{array}\right)\left(\begin{array}{r}
-3 \\
1 \\
-2
\end{array}\right)\left(\begin{array}{r}
-4 \\
2 \\
-2
\end{array}\right)\left(\begin{array}{r}
-4 \\
1 \\
-2
\end{array}\right)
\end{array}
$$


This leads to an invalid solution for the marking in each case. Thus, $\boldsymbol{m}^{*}$ is not reachable as a $\hat{\boldsymbol{m}}$-marking, too.

\section{Conclusion}

We have presented a new state equation for Timed Petri nets. In contrast to [2] the new equation is a holistic one: It describes the marking as well as the clocks, whereas [2] has dealt with the net's marking only.

Also, we have demonstrated in an example how the new state equation can be used to prove non-reachability.

\section{References}

1. Ramchandani, C.: Analysis of asynchronous concurrent systems by Timed Petri Nets. Project MAC-TR 120, MIT (February 1974)

2. Popova-Zeugmann, L., Werner, M., Richling, J.: Using state equation to prove non-reachability in timed petrinets. Fundam. Inf. 55(2) (August 2002) 187-202

3. Popova-Zeugmann, L., Pelz, E.: Algebraical characterisation of interval-timed petri nets with discrete delays. Fundamenta Informaticae 120(3-4) (2012) 341-357 